\title{
Association between Bone Mineral Density and Severity of Chronic Kidney Disease
}

\author{
Jin-Feng Huang, ${ }^{1}$ Xuan-Qi Zheng, ${ }^{1}$ Xiao-Lei Sun, ${ }^{2}$ Xiao Zhou, ${ }^{3}$ Jian Liu, ${ }^{4}$ Yan Michael Li, ${ }^{5}$ \\ Xiang-Yang Wang, ${ }^{1}$ Xiao-Lei Zhang $\mathbb{D}^{1},{ }^{1}$ and Ai-Min Wu $\mathbb{D}^{1}$ \\ ${ }^{1}$ Department of Orthopaedics, The Second Affiliated Hospital and Yuying Children's Hospital of Wenzhou Medical University, \\ The Second School of Medicine, Wenzhou Medical University, Wenzhou, Zhejiang 325027, China \\ ${ }^{2}$ Department of Orthopaedics, Tianjin Hospital, Tianjin, China \\ ${ }^{3}$ Ruian Institute of Quality and Technical Supervision and Inspection, Wenzhou 325000, China \\ ${ }^{4}$ Department of Nephrology, Rui Jin Hospital, Shanghai Jiao Tong University, School of Medicine, Shanghai 200025, China \\ ${ }^{5}$ Department of Neurosurgery and Oncology, University of Rochester Medical Center, School of Medicine and Dentistry, \\ Rochester, NY 14642, USA
}

Correspondence should be addressed to Xiao-Lei Zhang; zhangxiaolei@wmu.edu.cn and Ai-Min Wu; aiminwu@wmu.edu.cn

Received 22 July 2020; Revised 25 September 2020; Accepted 13 October 2020; Published 28 October 2020

Academic Editor: Silvia Monticone

Copyright (c) 2020 Jin-Feng Huang et al. This is an open access article distributed under the Creative Commons Attribution License, which permits unrestricted use, distribution, and reproduction in any medium, provided the original work is properly cited.

\begin{abstract}
Objective. We sought to evaluate the association between femoral neck (FN) and lumbar spine (LS) bone mineral densities (BMDs) with severity of chronic kidney disease (CKD) and prevalence of osteopenia or osteoporosis (OP) among the CKD group. Methods. Cross-sectional data from 11050 participants aged $\geq 20$ years from the National Health and Nutrition Examination Survey (NHANES) were analyzed. Specifically, Pearson correlation was applied to analyze the relationship between BMD and estimated glomerular filtration rate (eGFR). General linear models (GLMs) were adjusted for potential confounders and used to analyze mean BMD, based on CKD and CKD stages. Results. FN BMD was positively correlated with the eGFR in the total and male CKD, but not in the female CKD population. LS BMD was not significantly associated with eGFR. After controlling for partial correlations, FN T-score was positively correlated with the eGFR in the total at-risk population. According to FN BMD, OP prevalence was positively associated with CKD stage. However, according to LS BMD, there was no significant association between OP and CKD stage. Conclusion. Our results may explain the higher prevalence of hip fracture, relative to that of the spine, among CKD patients and generate meaningful insights to guide care, prevention, and treatment regimens for CKD patients. However, the fact that this was a cross-sectional study may limit the possibility of drawing concrete conclusions. Nevertheless, these findings open up a new frontier for further studies to uncover the higher decrease of FN BMD compared to LS BMD among CKD cases.
\end{abstract}

\section{Introduction}

Chronic kidney disease (CKD) is a common global public health problem [1-3]. The disease is defined when a person exhibits kidney damage or glomerular filtration rate (GFR) of $<60 \mathrm{ml} / \mathrm{min} / 1.73 \mathrm{~m}^{2}$ for 3 months or more [4]. Based on GFR and albuminuria, the National Kidney Foundation's Kidney Disease Outcomes Quality Initiative (KDOQI) has recommended classification of CKD into different stages [5], since patients at different stages of the disease exhibit distinct prognosis as well as body states.

Bone fractures are a common occurrence in patients with CKD [6,7]. For example, osteoporosis (OP), a systemic skeletal disease manifested as low bone mass and destruction of the bone tissue microenvironment, has been described [8]. In fact, OP patients always exhibit an increase in bone fragility and are more prone to fractures [9]. Although CKD and OP are two different diseases, they both negatively affect 
bone metabolism [10-12]. For instance, mineral and bone disorders were a common occurrence in CKD patients [13], leading to coining of the term of CKD-mineral and bone disorder (CKD-MBD) [14]. Generally, CKD-MBD means a systemic disorder containing one or combination of the following: (1) abnormalities in calcium, phosphorus, parathyroid hormone (PTH), and vitamin D metabolism; (2) calcification of vascular or other soft tissues; and/or (3) abnormalities in bone turnover, mineralization, or strength. These phenomena lead to bone disease and increased risk of fractures $[14,15]$.

Previous studies have shown that CKD occurrence can increase the risk of bone disease and fractures [16-18]. For example, CKD stages $3 \mathrm{a}-5 \mathrm{D}$ were found to have low bone mineral density (BMD) and 1.5 to 2-fold higher risk of fractures than in the general population [19]. However, conflicting results have been reported with regard to the complex association between BMD and kidney function [20-23], necessitating further studies.

The dual-energy X-ray absorptiometry (DXA) is a good predictor of fracture risk and the gold standard method for measuring BMD. In 2009, Kidney Disease: Improving Global Outcomes (KDIGO) guidelines were recommended for routine BMD testing [24]. However, these guidelines were updated in 2017 allowing their use in predicting the risk of fracture in CKD patients [25]. This change of guidelines indicates that using $\mathrm{BMD}$ and $\mathrm{T}$-score screening for osteopenia and OP can be used for CKD patients.

Prevalence of osteopenia and OP, as well as the association between BMD and renal function among American CKD patients, is not well understood [26]. In the current study, we thoroughly analyze the association, adjusted for multiple potential confounders, between BMD and eGFR among American CKD patients. We used many statistical methods such as general liner model, Pearson correlation, and partial correlations to assess the relationship. Moreover, we also included multiple potential confounders, especially medication taken history, into analysis. Besides, the large number of patients $(>10,000)$ studied constitute one of the main strengths of our analysis. The statistical power of our study was therefore relatively high.

\section{Methods}

2.1. Data Sources and Study Participants. In this study, we collected data from the National Health and Nutrition Examination Survey (NHANES) database. The NHANES, conducted by the National Center for Health Statistics (NCHS), aims to assess the health and nutritional status of the US population [27]. Prior to data collection, all NHANES survey protocols are required to be evaluated by the NCHS Research Ethics Board, and informed consent was signed [28]. The present study extracted data from four NHANES cycles, including the periods between 2005-2010 and 20132014. In these cycles, BMDs of the femoral neck and lumbar spine were measured by DXA. For this study, no ethical approval was required, since we analyzed public data.

The analytic cohorts were derived from adults, aged 20 years and older, in NHANES. Respondents who lacked valid FN BMD and LS BMD data or did not have data for at least two lumbar vertebrae were excluded from the analysis [29].

2.2. Retrieval of Clinical, Laboratory, and BMD Data. We retrieved demographic and comorbidity data, including age, sex, BMI, diabetes, coronary heart disease, arthritis, congestive heart failure, stroke, chronic bronchitis, and smoking history, as well as laboratory findings (e.g., albumin, alkaline phosphatase (ALP), calcium, creatinine, phosphorus, vitamin D, and glycohemoglobin levels) according to previous studies $[30,31]$. Other variables collected included fracture history (hip, wrist, spine, and other sites), menopause status, status, and types of medication taken such as antiresorptive drugs (e.g., alendronate, risedronate, ibandronate, raloxifene, and calcitonin), angiotensin-converting enzyme inhibitors (ACEI), estrogen, loop diuretics, and glucocorticoids, as well as research year cycles and DXA results.

2.3. Measurement and Interpretation of Bone Mineral Densities. In NHANES 2005-2010, femoral neck and posterior-anterior lumbar spine scans were examined using Hologic QDR 4500A fan-beam, while those from 2013 to 2014 were measured by Hologic Discovery ${ }^{\circledR}$ A (Hologic Inc., Marlborough, MA) densitometers [32]. In the 2005-2010 segment, Hologic Discovery v12.4 was used for analyzing femur scans, whereas the spine scans were assessed using APEX v3.0. On the other hand, both femur and spine scans in the 2013-2014 segment were analyzed using APEX v4.0. Previous studies have revealed no significant differences in mean BMDs analyzed using Hologic Discovery v12.4 and APEX v4.0 [33].

Studies have also indicated that male subjects, subjects older than 50 years, and postmenopausal females exhibit marked bone mineral loss; therefore, this population is more prone to osteoporotic fracture $[34,35]$. Based on this, we defined male participants aged $\geq 50$ and postmenopausal women as at-risk population. For this population, we calculated T-scores before analyzing the association between OP prevalence and CKD stages. T-scores were defined as BMD respondent minus the mean BMD of the reference group and then divided by standard deviation (SD) of the reference group. We considered 30-year-old white females from the DXA manufacturer reference database, and 20-29-year-old non-Hispanic white females, from NHANES III, as the reference groups for lumbar spine and femoral neck, respectively [36]. In addition, we defined osteopenia as a $\mathrm{T}$-score between -1.0 and -2.5 and $\mathrm{OP}$ as a $\mathrm{T}$-score $\leq-2.5$ according to the criteria recommended by the World Health Organization (WHO) $[37,38]$. We also defined "low T-score" as having the lowest T-score $\leq-1$, that is, within the range of osteopenia and OP. So, using low T-score can find low bone mass patients more sensitive. OP prevalence and low bone mass were calculated separately, for femur neck and lumbar spine. 
2.4. Measurement of Chronic Kidney Disease. To calculate estimated glomerular filtration rate (eGFR), we adopted the Chronic Kidney Disease Epidemiology Collaboration equation [39]. Briefly, albuminuria was defined as the ratio of urinary albumin to creatinine (ACR) [40]. CKD was defined as ACR $\geq 30 \mathrm{mg} / \mathrm{g}$ or eGFR blow $60 \mathrm{~mL} / \mathrm{min} / 1.73 \mathrm{~m}^{2}$ [41]. We divided CKD participants into different stages, according to the guidelines provided by the KDIGO for further study [5]. Stage $1 \mathrm{CKD}$ was defined as cases with eGFR $\geq 90 \mathrm{~mL} / \mathrm{min} / 1.73 \mathrm{~m}^{2}$ and ACR $\geq 30 \mathrm{mg} / \mathrm{g}$, stage $2 \mathrm{CKD}$ as eGFR 60 to $89 \mathrm{~mL} / \mathrm{min} / 1.73 \mathrm{~m}^{2}$ and ACR $\geq 30 \mathrm{mg} / \mathrm{g}$, stage 3 CKD as eGFR 30 to $59 \mathrm{~mL} / \mathrm{min} / 1.73 \mathrm{~m}^{2}$, and stage 4 as those with eGFR $<30 \mathrm{~mL} / \mathrm{min} / 1.73 \mathrm{~m}^{2}$.

2.5. Anthropometric Measurement. Weight (kg) and height $(\mathrm{cm})$ of the respondents were measured while they were dressed in light clothing without shoes and their BMI defined as the ratio of weight to the square of the height $(\mathrm{kg} /$ $\mathrm{m}^{2}$ ). Thereafter, their BMIs were divided into three groups $(<25,25$ to $<30, \geq 30)$, whereas smoking history was classified into two groups ( $<100$ cigarettes and $\geq 100$ cigarettes).

2.6. Statistical Analysis. Four NHANES cycles (2005-2006, 2007-2008, 2009-2010, and 2013-2014) were pooled before analysis. Participant characteristics were first compared between CKD and the control group. All continuous variables evaluated their distribution firstly. For normal variable distribution variables, we conducted Student's $t$-test, whereas nonnormal variable distribution ones were subjected to the nonparametric tests. The categorical variables were compared using the Chi-square test. Characteristics of participants among subgroups at different CKD stages were compared using analysis of variance (ANOVA), for continuous variables, and the Chi-square test for categorical ones.

A Pearson correlation matrix was used to analyze the relationship between BMD and eGFR. Another Pearson correlation analysis was also done after classifying different groups according to sex to investigate specific associations. We then performed partial correlations, while controlling for race, age, and sex (partial correlation 1) and race, age, sex, and BMI (partial correlation 2) and for all covariates (age, sex, BMI, ALP, albumin, total calcium, phosphorus, 25(OH) D3, glycohemoglobin, diabetes, coronary heart disease, arthritis, congestive heart failure, stroke and chronic bronchitis, fracture history, menopause status, medication, and research year cycles) (partial correlation 3). During genderbased subgroup analysis, we excluded sex variate from controlling analysis.

The relationships between BMD and CKD stage were further analyzed in patients, using generalized linear models (GLM), with adjustment for multiple covariates. Age, sex, and BMI were considered essential covariates, owing to their marked association with CKD and OP [42-45]. Model 1 was adjusted by age, sex, and race and Model 2 by age, sex, race, and BMI, whereas Model 3 was adjusted for age, sex, BMI, ALP, albumin, total calcium, phosphorus, 25(OH)D3, glycohemoglobin, diabetes, coronary heart disease, arthritis, congestive heart failure, stroke, and chronic bronchitis, as well as fracture history, menopause status, medication, and research year cycles. During sex-based subgroup analyses, we excluded gender from model controlling. The multicollinearities between covariates were evaluated by correlation analyses and collinearity statistics. Post hoc analyses were conducted using GLM or logistic regression setting, with stage 1 CKD taken as the reference subgroup.

All statistical analyses were performed in SPSS software (version 18; IBM Corp., USA). Since multiple testing may result in type I error, we considered $P<0.01$ as statistically significant during investigations of several endpoints or performed several statistical tests on the same data, such as ANOVA and post hoc analyses [46]. We considered $P<0.05$ to be statistically significant across results from Student's $t$ tests and Chi-square, GLM, and logistic regression model.

\section{Results}

3.1. Relationship between BMD and the Presence of CKD. A total of 11,050 respondents, 1,572 with and 9,478 without CKD were recruited in our study between 2005-2010 and 2013-2014. A summary of their characteristics is outlined in Table 1. The mean FN BMD was lower in the CKD group compared to that without $(P<0.001)$, whereas no significant difference was observed between the groups with regard to LS BMD $(P=0.114)$.

OP prevalence for FN BMD was significantly higher in the CKD group (7.2\%) compared to the group without (3.0\%). In addition, prevalence of OP calculating for FN BMD was more common in female CKD patients $(12.3 \%)$. Conversely, OP prevalence for LS BMD revealed no significant differences between the two groups. A detailed description for prevalence of osteopenia, OP, and low T-scores across the 2 groups is outlined in Table 2 .

3.2. Association between $B M D$ and eGFR. We further investigated the relationship between low BMD and low eGFR. Scatterplots and Pearson correlation coefficients revealed a positive correlation between FN BMD and eGFR in the CKD population $(r=0.221 ; P<0.0001$ in the total population; $r=0.303 ; P<0.0001$ in the female population; $r=0.266$; $P<0.0001$ in the male population; Figures $1(\mathrm{a})-1(\mathrm{c}))$. However, there is no significant relationship between LS BMD and eGFR $(r=0.021 ; P=0.402$ in the total population; Figures 1(d) and 1(f)). After performing partial correlations to control for race, age, and sex (partial correlation 1); race, age, sex, and BMI (partial correlation 2); and all covariates (partial correlation 3), FN BMD was positively correlated with the eGFR in the total and male CKD populations, but not in female CKD population (Figure $1(\mathrm{~g})$ ).

\subsection{Association between BMD and CKD Severity. Mean FN} and LS BMDs according to subgroup by CKD stage are presented in Table 3. Summarily, unadjusted and adjusted FN BMDs were significantly different between subgroups based on the CKD stage. However, no significant differences were obtained for LS BMD (Table 3). Post hoc analyses 
TABLE 1: Comparison of sample characteristics between those with chronic kidney disease according to current chronic kidney disease status.

\begin{tabular}{|c|c|c|c|c|c|c|c|c|}
\hline & $\begin{array}{l}\text { Healthy } \\
(N=9478)\end{array}$ & $\begin{array}{l}\text { CKD group } \\
(N=1572)\end{array}$ & $\begin{array}{c}P \\
\text { value }^{1}\end{array}$ & $\begin{array}{c}\text { Stage I } \\
(N=532)\end{array}$ & $\begin{array}{c}\text { Stage II } \\
(N=381)\end{array}$ & $\begin{array}{l}\text { Stage III } \\
(N=597)\end{array}$ & $\begin{array}{l}\text { Stage IV } \\
(N=62)\end{array}$ & $\begin{array}{c}P \\
\text { value }^{2} \\
\end{array}$ \\
\hline $\begin{array}{l}\text { Age, years } \\
(\text { mean } \pm S D)\end{array}$ & $45.71 \pm 15.38$ & $60.42 \pm 16.68$ & $<0.001$ & $45.44 \pm 13.68$ & $63.38 \pm 13.48$ & $71.18 \pm 10.39$ & $67.11 \pm 12.68$ & $<0.0001$ \\
\hline $\begin{array}{l}e G F R(m L / \\
\left.m i n / 1.73 m^{2}\right)\end{array}$ & $98.21 \pm 18.01$ & $74.76 \pm 28.79$ & $<0.001$ & $108.18 \pm 11.88$ & $76.03 \pm 8.67$ & $50.07 \pm 7.63$ & $17.90 \pm 8.69$ & $<0.0001$ \\
\hline $\begin{array}{l}\mathrm{BMI}, \mathrm{kg} / \mathrm{m}^{2} \\
(\mathrm{mean} \pm \mathrm{SD})\end{array}$ & $27.99 \pm 5.55$ & $28.69 \pm 5.94$ & $<0.001$ & $29.14 \pm 6.85$ & $28.64 \pm 5.62$ & $28.36 \pm 5.25$ & $28.22 \pm 5.54$ & 0.148 \\
\hline$F N B M D\left(\mathrm{~g} / \mathrm{cm}^{2}\right)$ & $0.96 \pm 0.17$ & $0.91 \pm 0.18$ & $<0.0001$ & $0.96 \pm 0.17$ & $0.90 \pm 0.19$ & $0.88 \pm 0.17$ & $0.82 \pm 0.19$ & $<0.0001$ \\
\hline$L S B M D\left(\mathrm{~g} / \mathrm{cm}^{2}\right)$ & $1.03 \pm 0.15$ & $1.03 \pm 0.18$ & 0.114 & $1.04 \pm 0.15$ & $1.02 \pm 0.18$ & $1.02 \pm 0.19$ & $1.05 \pm 0.20$ & 0.227 \\
\hline $\begin{array}{l}\text { Laboratorial test } \\
\text { Albumin }(\mathrm{g} / \mathrm{dl})\end{array}$ & $4.27 \pm 0.31$ & $4.16 \pm 0.34$ & $<0.001$ & $4.19 \pm 0.36$ & $4.19 \pm 0.33$ & $4.14 \pm 0.31$ & $3.93 \pm 0.49$ & $<0.0001$ \\
\hline $\begin{array}{l}\text { Alkaline phosphatase } \\
(\mathrm{U} / \mathrm{L})\end{array}$ & $68.11 \pm 22.68$ & $74.96 \pm 31.41$ & $<0.001$ & $75.48 \pm 38.03$ & $75.83 \pm 27.45$ & $71.91 \pm 25.13$ & $94.47 \pm 38.26$ & $<0.0001$ \\
\hline $\begin{array}{l}\text { Total calcium } \\
(\mathrm{mg} / \mathrm{dL})\end{array}$ & $9.45 \pm 0.35$ & $9.46 \pm 0.41$ & 0.424 & $9.44 \pm 0.39$ & $9.51 \pm 0.40$ & $9.46 \pm 0.39$ & $9.26 \pm 0.55$ & $<0.0001$ \\
\hline Phosphorus (mg/dL) & $3.76 \pm 0.55$ & $3.77 \pm 0.62$ & 0.724 & $3.71 \pm 0.56$ & $3.69 \pm 0.57$ & $3.77 \pm 0.56$ & $4.63 \pm 1.13$ & $<0.0001$ \\
\hline $25(\mathrm{OH}) \mathrm{D} 3$ & 26.02 & & 0.123 & & & & & 0.248 \\
\hline Glycohemoglobin, \% & $5.56 \pm 0.84$ & & $<0.001$ & $6.34 \pm 1,96$ & & $6.01 \pm 1.05$ & $6.26 \pm 1.69$ & 0.01 \\
\hline $\begin{array}{l}\text { Gender }(n, \%) \\
\text { Male } \\
\text { Female }\end{array}$ & $\begin{array}{l}4782(50.5 \%) \\
4696(49.5 \%) \\
\end{array}$ & $\begin{array}{l}757(48.2 \%) \\
815(51.8 \%) \\
\end{array}$ & 0.091 & $\begin{array}{l}205(38.5 \%) \\
327(61.5 \%) \\
\end{array}$ & $\begin{array}{l}197(51.7 \%) \\
184(48.3 \%) \\
\end{array}$ & $\begin{array}{l}324(54.3 \%) \\
273(45.7 \%) \\
\end{array}$ & $\begin{array}{l}31(50.0 \%) \\
31(50.0 \%) \\
\end{array}$ & $<0.0001$ \\
\hline $\begin{array}{l}\text { Race }(n, \%) \\
\text { Non-Hispanic white } \\
\text { Non-Hispanic black } \\
\text { Mexican-American } \\
\text { Other Hispanic } \\
\text { Other race }\end{array}$ & $\begin{aligned} & 1915(20.2 \%) \\
& 903(9.5 \%) \\
& 4486(47.3 \%) \\
& 1743(18.4 \%) \\
& 431(4.5 \%) \\
&\end{aligned}$ & $\begin{array}{c}274(17.4 \%) \\
132(8.4 \%) \\
768(48.9 \%) \\
343(21.8 \%) \\
55(3.5 \%)\end{array}$ & $<0.001$ & $\begin{array}{c}157(29.5 \%) \\
58(10.9 \%) \\
170(32.0 \%) \\
120(22.6 \%) \\
27(5.1 \%)\end{array}$ & $\begin{array}{c}62(16.3 \%) \\
35(9.2 \%) \\
187(49.1 \%) \\
83(21.8 \%) \\
14(3.6 \%)\end{array}$ & $\begin{aligned} 50 & (8.4 \%) \\
37 & (6.2 \%) \\
389 & (65.2 \%) \\
109 & (18.3 \%) \\
12 & (2.0 \%)\end{aligned}$ & $\begin{aligned} & 5(8.1 \%) \\
& 2(3.2 \%) \\
& 22(35.5 \%) \\
& 31(50.0 \%) \\
& 2(3.2 \%) \\
&\end{aligned}$ & $<0.0001$ \\
\hline \multicolumn{9}{|l|}{$\begin{array}{l}\text { Fracture history } \\
(n, \%)\end{array}$} \\
\hline Previous hip fracture & $100(1.1 \%)$ & $31(2.0 \%)$ & 0.008 & $8(1.5 \%)$ & $10(2.6 \%)$ & $13(2.2 \%)$ & - & 0.615 \\
\hline $\begin{array}{l}\text { Previous wrist } \\
\text { fracture }\end{array}$ & $825(8.7 \%)$ & $156(9.9 \%)$ & 0.290 & $39(7.3 \%)$ & $44(11.5 \%)$ & $66(11.1 \%)$ & $7(11.3 \%)$ & 0.159 \\
\hline $\begin{array}{l}\text { Previous spine } \\
\text { fracture }\end{array}$ & $150(1.6 \%)$ & $29(1.8 \%)$ & 0.510 & $8(1.5 \%)$ & $7(1.8 \%)$ & $14(2.3 \%)$ & - & 0.714 \\
\hline $\begin{array}{l}\text { Previous other site } \\
\text { fractures }\end{array}$ & $1940(20.5 \%)$ & $368(23.4 \%)$ & 0.025 & $96(18.0 \%)$ & $97(25.5 \%)$ & $157(26.3 \%)$ & $18(29.0 \%)$ & 0.022 \\
\hline $\begin{array}{l}\text { Lifetime smoking } \\
(n, \%)\end{array}$ & & & 0.011 & & & & & 0.247 \\
\hline$<100$ cigarettes & $4314(45.5 \%)$ & $770(49.0 \%)$ & & $246(46.2 \%)$ & & & $36(58.1 \%)$ & \\
\hline$\geq 100$ cigarettes & $5164(54.5 \%)$ & $802(51.0 \%)$ & & $286(53.8 \%)$ & $194(50.9 \%)$ & $296(49.6 \%)$ & $26(41.9 \%)$ & \\
\hline Menopause ( $n, \%)$ & $1731(18.3 \%)$ & $506(32.2 \%)$ & $<0.001$ & $98(18.4 \%)$ & $131(34.4 \%)$ & $254(42.5 \%)$ & $23(37.1 \%)$ & $<0.0001$ \\
\hline \multicolumn{9}{|l|}{ Medication ( $n, \%)$} \\
\hline Antiresorptive drugs & $103(1.1 \%)$ & & $<0.001$ & & & & $1(1.6 \%)$ & $<0.0001$ \\
\hline ACEI & $815(8.6 \%)$ & $391(24.9 \%)$ & $<0.001$ & $78(14.7 \%)$ & $106(27.8 \%)$ & $188(31.5 \%)$ & $19(30.6 \%)$ & $<0.0001$ \\
\hline Estrogen & $91(1.0 \%)$ & $18(1.1 \%)$ & 0.892 & $1(0.2 \%)$ & $6(1.6 \%)$ & $10(1.7 \%)$ & $1(1.6 \%)$ & $<0.0001$ \\
\hline & $95(1.0 \%)$ & & $<0.001$ & & & & $21(33.9 \%)$ & $<0.0001$ \\
\hline Glucocorticoids & $381(4.0 \%)$ & $108(6.9 \%)$ & $<0.001$ & $28(5.3 \%)$ & $18(4.7 \%)$ & $52(8.7 \%)$ & $10(16.1 \%)$ & $<0.0001$ \\
\hline \multicolumn{9}{|l|}{$\begin{array}{l}\text { Known comorbidities } \\
(n, \%)\end{array}$} \\
\hline Diabetes mellitus & $645(6.8 \%)$ & $399(25.4 \%)$ & $<0.001$ & $125(23.5 \%)$ & $113(29.7 \%)$ & $132(22.1 \%)$ & $29(46.8 \%)$ & $<0.0001$ \\
\hline $\begin{array}{l}\text { Coronary heart } \\
\text { disease }\end{array}$ & $206(2.2 \%)$ & $141(9.0 \%)$ & $<0.001$ & $13(2.4 \%)$ & $43(11.3 \%)$ & $69(11.6 \%)$ & $16(25.8 \%)$ & $<0.0001$ \\
\hline Arthritis & $1995(21.0 \%)$ & $570(36.3 \%)$ & $<0.001$ & $112(21.1 \%)$ & $146(38.3 \%)$ & $281(47.1 \%)$ & $31(50.0 \%)$ & $<0.001$ \\
\hline $\begin{array}{l}\text { Congestive heart } \\
\text { failure }\end{array}$ & $89(0.9 \%)$ & 74 (4.7\%) & $<0.001$ & $9(1.7 \%)$ & $24(6.3 \%)$ & $31(5.2 \%)$ & $10(16.1 \%)$ & $<0.0001$ \\
\hline Stroke & $176(1.9 \%)$ & $113(7.2 \%)$ & $<0.001$ & & $28(7.3 \%)$ & & $14(22.6 \%)$ & $<0.0001$ \\
\hline Chronic bronchitis & $471(5.0 \%)$ & $129(8.2 \%)$ & $<0.001$ & $32(6.0 \%)$ & $31(8.1 \%)$ & $59(9.9 \%)$ & $7(11.3 \%)$ & 0.061 \\
\hline
\end{tabular}


TABLE 1: Continued.

\begin{tabular}{|c|c|c|c|c|c|c|c|c|}
\hline & $\begin{array}{l}\text { Healthy } \\
(N=9478)\end{array}$ & $\begin{array}{c}\text { CKD group } \\
(N=1572)\end{array}$ & $\begin{array}{c}P \\
\text { value }^{1}\end{array}$ & $\begin{array}{c}\text { Stage I } \\
(N=532)\end{array}$ & $\begin{array}{c}\text { Stage II } \\
(N=381)\end{array}$ & $\begin{array}{l}\text { Stage III } \\
(N=597)\end{array}$ & $\begin{array}{l}\text { Stage IV } \\
(N=62)\end{array}$ & $\begin{array}{c}P \\
\text { value }^{2}\end{array}$ \\
\hline \multicolumn{9}{|c|}{ Year cycles (n,\%) } \\
\hline 2005-2006 & $2368(25.0 \%)$ & 447 (28.4\%) & $<0.001$ & $126(23.7 \%)$ & $97(25.5 \%)$ & 206 (34.5\%) & $18(29.0 \%)$ & $<0.0001$ \\
\hline 2007-2008 & $2662(28.1 \%)$ & $492(31.3 \%)$ & $<0.001$ & $180(33.8 \%)$ & $113(29.7 \%)$ & $183(30.7 \%)$ & $16(25.8 \%)$ & 0.384 \\
\hline 2009-2010 & $3068(32.4 \%)$ & $389(24.7 \%)$ & $<0.001$ & $143(26.9 \%)$ & $89(23.4 \%)$ & $143(24.0 \%)$ & $14(22.6 \%)$ & 0.562 \\
\hline 2013-2014 & $1380(14.6 \%)$ & $244(15.5 \%)$ & 0.319 & $83(15.6 \%)$ & $82(21.5 \%)$ & $65(10.9 \%)$ & $14(22.6 \%)$ & $<0.0001$ \\
\hline
\end{tabular}

Values are the means \pm SE or $n(\%)$, as appropriate. $P$ values ${ }^{1}$ by Student's $t$ tests or nonparametric tests for continuous variables and the Chi-square test for categorical variables; $P<0.05$ was considered statistically significant. $P$ values ${ }^{2}$ by analysis of variance (ANOVA) for continuous variables and the Chisquared test for categorical variables; $P<0.01$ was considered statistically significant. FN, femoral neck; LS, lumbar spine; BMD, bone mineral density; CKD: chronic kidney disease; NHANES: the National Health and Nutrition Examination Survey; eGFR: estimated glomerular filtration rate; 25(OH)D3, 25hydroxyvitamin D3.

TABLE 2: Prevalence of osteopenia, osteoporosis, or low t-score in the at-risk population according to the presence of CKD (male participants aged $\geq 50$ years and postmenopausal women).

\begin{tabular}{|c|c|c|c|}
\hline & Healthy $(N=3577)$ & CKD group $(N=1104)$ & $P$ value \\
\hline \multicolumn{4}{|l|}{ Femoral neck } \\
\hline \multicolumn{4}{|l|}{ Total population } \\
\hline Osteopenia $(n, \%)$ & $1076(30.1 \%)$ & $382(34.6 \%)$ & $<0.001$ \\
\hline Osteoporosis $(n, \%)$ & $108(3.0 \%)$ & $79(7.2 \%)$ & 0.005 \\
\hline Low T-score $(n, \%)$ & $1184(33.1 \%)$ & $461(41.8 \%)$ & $<0.001$ \\
\hline \multicolumn{4}{|l|}{ Female } \\
\hline Osteopenia $(n, \%)$ & $656(37.9 \%)$ & $214(42.3 \%)$ & 0.074 \\
\hline Osteoporosis $(n, \%)$ & $91(5.3 \%)$ & $62(12.3 \%)$ & $<0.001$ \\
\hline Low T-score $(n, \%)$ & $747(43.2 \%)$ & $276(54.5 \%)$ & $<0.001$ \\
\hline \multicolumn{4}{|l|}{ Male } \\
\hline Osteopenia $(n, \%)$ & $420(22.8 \%)$ & $168(28.1 \%)$ & 0.008 \\
\hline Osteoporosis $(n, \%)$ & $17(0.9 \%)$ & $17(2.8 \%)$ & $<0.001$ \\
\hline Low T-score $(n, \%)$ & $437(23.7 \%)$ & $185(30.9 \%)$ & $<0.001$ \\
\hline \multicolumn{4}{|l|}{ Lumbar spine } \\
\hline \multicolumn{4}{|l|}{ Total population } \\
\hline Osteopenia $(n, \%)$ & $1064(29.7 \%)$ & $274(24.8 \%)$ & 0.063 \\
\hline Osteoporosis $(n, \%)$ & $254(7.1 \%)$ & $97(8.8 \%)$ & 0.002 \\
\hline Low T-score $(n, \%)$ & $1318(36.8 \%)$ & $371(33.6 \%)$ & $<0.05$ \\
\hline \multicolumn{4}{|l|}{ Female } \\
\hline Osteopenia $(n, \%)$ & $661(38.2 \%)$ & $176(34.8 \%)$ & 0.164 \\
\hline Osteoporosis $(n, \%)$ & $200(11.6 \%)$ & $81(16.0 \%)$ & 0.008 \\
\hline Low T-score $(n, \%)$ & $861(49.7 \%)$ & $257(50.8 \%)$ & 0.678 \\
\hline \multicolumn{4}{|l|}{ Male } \\
\hline Osteopenia $(n, \%)$ & $403(21.8 \%)$ & $98(16.4 \%)$ & 0.004 \\
\hline Osteoporosis $(n, \%)$ & $54(2.9 \%)$ & $16(2.7 \%)$ & 0.750 \\
\hline Low T-score $(n, \%)$ & $457(24.8 \%)$ & $114(19.1 \%)$ & 0.004 \\
\hline
\end{tabular}

$P$ values by the Chi-square test; $P<0.05$ was considered statistically significant. The at-risk population, male participants aged $\geq 50$ years, and postmenopausal women; low T-score and T-score $\leq-1$.

showed that unadjusted FN BMD in CKD stages 2, 3, and 4 were significantly different with CKD stage 1 . After adjusting for race, age, and sex, FN BMD still showed a negative relationship with $\mathrm{CKD}$ stage, although LS BMD had a positive relationship with CKD stage (Model 1 for FN and LS BMDs in Table 3). Further adjusting for BMI and other covariates revealed similar results, with a negative relationship between FN BMD and CKD stage as well as a positive correlation between LS BMD and CKD stage (Models 2 and 3 for FN and LS BMDs in Table 3). Moreover, the relationship between LS BMD and CKD stage remained insignificant even after adjusting for covariates (Models 1, 2, and 3 for LS BMD in Table 3 ).
3.4. Association between T-Score and eGFR in the At-Risk Population. We further investigated whether low T-scores were associated with low eGFR in the at-risk population. Scatter plots and Pearson correlation coefficients revealed a positive correlation between FN T-score and eGFR in the at-risk population (Figures 2(a)-2(c)). However, there is no significant relationship between LS T-score and eGFR in female or male subgroup $(r=-0.026 ; P=0.565$ in the female at-risk population; $r=-0.069 ; P=0.091$ in the male at-risk population; Figures 2(e) and 2(f)) After controlling for partial correlations, we observed a positive correlation between FN T-score and the eGFR in the total at-risk population and a negative relationship between LS T-score and eGFR (Figure 2(g)). 

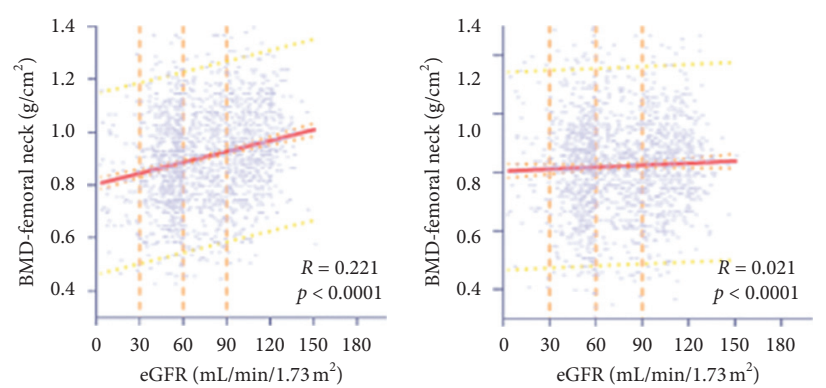

(a)
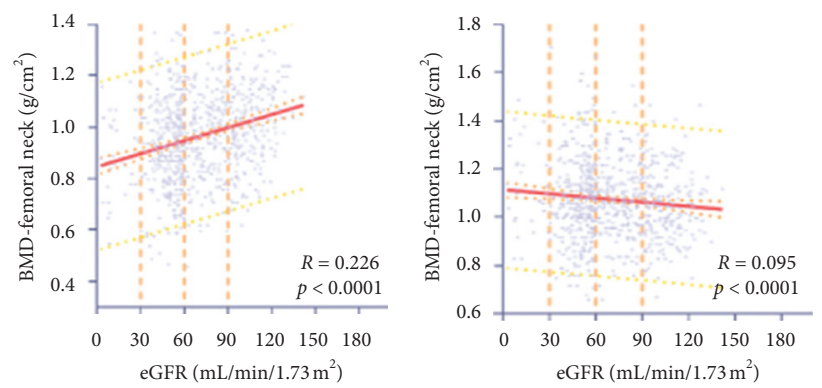

(b)

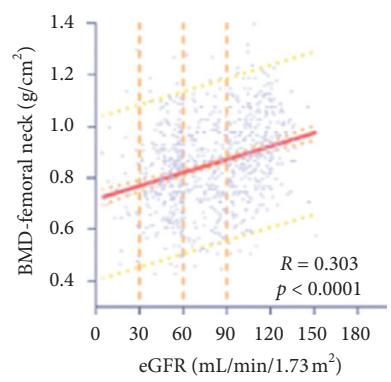

\begin{tabular}{|c|c|c|c|c|}
\hline \multirow[t]{2}{*}{ Total population } & \multicolumn{2}{|c|}{ Femoral neck } & \multicolumn{2}{|c|}{ Lumbar spine } \\
\hline & Coefficient & $P$ value & Coefficient & $P$ value \\
\hline Simple correlation & 0.221 & $<0.0001$ & 0.021 & 0.402 \\
\hline Partial correlation 1 & 0.031 & 0.309 & -0.048 & 0.145 \\
\hline Partial correlation 2 & 0.049 & 0.084 & -0.034 & 0.282 \\
\hline Partial correlation 3 & 0.068 & 0.015 & -0.043 & 0.178 \\
\hline
\end{tabular}

\begin{tabular}{lcccc}
\hline \multirow{2}{*}{ Male } & \multicolumn{2}{c}{ Femoral Neck } & \multicolumn{2}{c}{ Lumbar Spine } \\
& Coefficient & $P$ value & Coefficient & $P$ value \\
\hline Simple correlation & 0.266 & $<0.0001$ & -0.095 & 0.009 \\
Partial correlation 1 & 0.128 & 0.005 & -0.043 & 0.359 \\
Partial correlation 2 & 0139 & 0.001 & -0.035 & 0.439 \\
Partial correlation 3 & 0.125 & 0.006 & -0.053 & 0.296 \\
\hline
\end{tabular}

FIGURE 1: Scatterplot, Pearson correlation, and partial correlations between BMD and eGFR in total population, males, and females (a, b, and c, respectively). BMD is presented by a logarithmic scale along the $y$-axis and eGFR along the $x$-axis. Regression lines are shown with 95\% CIs (orange lines) and 95\% prediction interval (yellow lines). BMD, bone mineral density; CKD: chronic kidney disease; eGFR: estimated glomerular filtration rate.

TABLE 3: Mean BMD $\left(\mathrm{g} / \mathrm{cm}^{2}\right)$ according to the severity of CKD.

\begin{tabular}{|c|c|c|c|c|c|}
\hline & Stage I $(N=532)$ & Stage II $(N=381)$ & Stage III $(N=597)$ & Stage IV $(N=62)$ & $P$ for trend \\
\hline \multicolumn{6}{|c|}{ Femoral neck BMD } \\
\hline Unadjusted & $0.96 \pm 0.01$ & $0.90 \pm 0.01^{\dagger}$ & $0.88 \pm 0.01^{\dagger}$ & $0.82 \pm 0.02^{\dagger}$ & $<0.001$ \\
\hline Model 1 & $0.94 \pm 0.01$ & $0.90 \pm 0.01^{*}$ & $0.90 \pm 0.01^{*}$ & $0.84 \pm 0.02^{\dagger}$ & 0.002 \\
\hline Model 2 & $0.93 \pm 0.01$ & $0.90 \pm 0.01^{*}$ & $0.90 \pm 0.01^{*}$ & $0.84 \pm 0.02^{\dagger}$ & 0.006 \\
\hline Model 3 & $0.94 \pm 0.01$ & $0.91 \pm 0.01^{*}$ & $0.90 \pm 0.01^{*}$ & $0.86 \pm 0.03^{\dagger}$ & 0.033 \\
\hline \multicolumn{6}{|c|}{ Lumbar spine BMD } \\
\hline Unadjusted & $1.04 \pm 0.01$ & $1.02 \pm 0.01$ & $1.02 \pm 0.01$ & $1.05 \pm 0.02$ & 0.227 \\
\hline Model 1 & $1.02 \pm 0.01$ & $1.02 \pm 0.01$ & $1.03 \pm 0.01$ & $1.05 \pm 0.02$ & 0.727 \\
\hline Model 2 & $1.03 \pm 0.01$ & $1.02 \pm 0.01$ & $1.03 \pm 0.01$ & $1.05 \pm 0.02$ & 0.714 \\
\hline Model 3 & $0.99 \pm 0.01$ & $0.98 \pm 0.01$ & $0.98 \pm 0.01$ & $0.98 \pm 0.03$ & 0.917 \\
\hline
\end{tabular}

Values are the means \pm SE. $P$ for trend values by general linear models; $P<0.05$ was considered statistically significant. ${ }^{*} P<0.01$ and ${ }^{\dagger} P<0.001$ by $p o s t$ hoc analyses, CKD stage 1 subgroup as reference, and $P<0.01$ was considered statistically significant. Model 1 , adjusted for age, sex, and race. Model 2 , adjusted for age, sex, race, and BMI. Model 3, adjusted for age, sex, BMI, ALP, total calcium, albumin, phosphorus, 25(OH)D3, glycohemoglobin, diabetes, coronary heart disease, arthritis, congestive heart failure, stroke and chronic bronchitis, fracture history, menopause status, medication, and research year cycle. BMD, bone mineral density; CKD, chronic kidney disease; 25(OH)D3, 25-hydroxyvitamin D3; BMI, body mass index; ALP, alkaline phosphatase. 

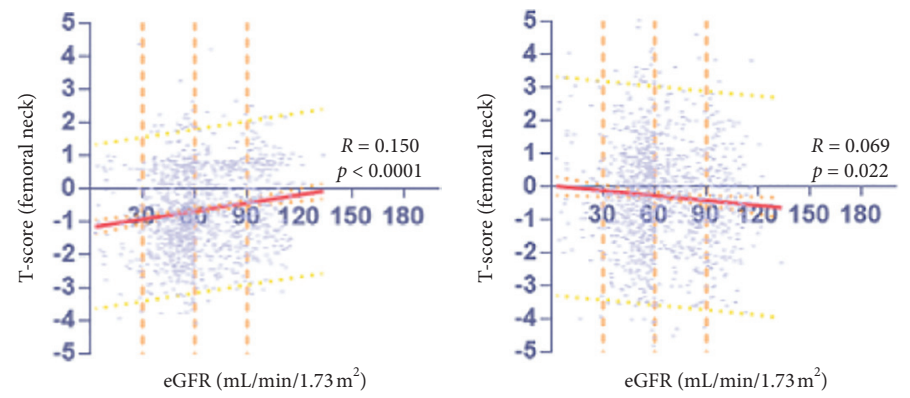

\begin{tabular}{lcccc}
\hline \multirow{2}{*}{ Total at-risk population } & \multicolumn{3}{c}{ T-score-femoral Neck } & \multicolumn{2}{c}{ T-score-lumbar spine } \\
& Coefficient & $P$ value & Coefficient & $P$ value \\
\hline Simple correlation & 0.15 & $<0.0001$ & 0.069 & 0.022 \\
Partial correlation 1 & 0.056 & 0.067 & -0.048 & 0.149 \\
Partial correlation 2 & 0.074 & 0.01 & -0.034 & 0.29 \\
Partial correlation 3 & 0.061 & 0.03 & -0.047 & 0.144 \\
\hline
\end{tabular}

(a)
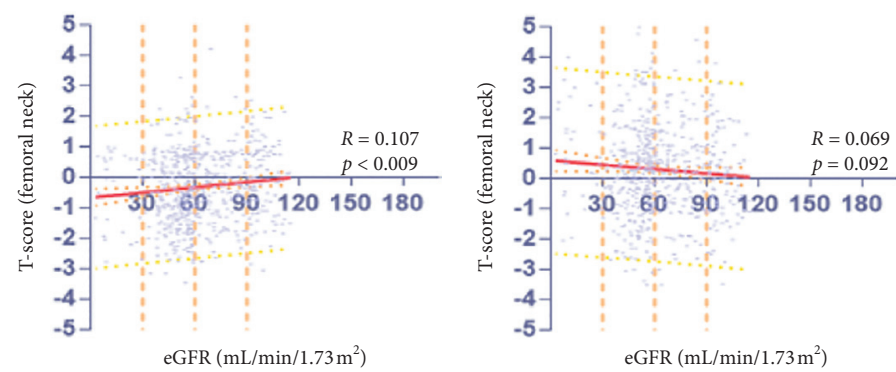

\begin{tabular}{lcccc}
\hline \multirow{2}{*}{ Male at-risk population } & \multicolumn{2}{c}{ T-score-femoral neck } & \multicolumn{2}{c}{ T-score-lumbar spine } \\
& Coefficient & $P$ value & Coefficient & $P$ value \\
\hline Simple correlation & 0.107 & 0.009 & -0.069 & 0.091 \\
Partial correlation 1 & 0.017 & 0.693 & -0.012 & 0.788 \\
Partial correlation 2 & 0.022 & 0.601 & -0.032 & 0.474 \\
Partial correlation 3 & 0.052 & 0.221 & -0.056 & 0.234 \\
\hline
\end{tabular}

(b)
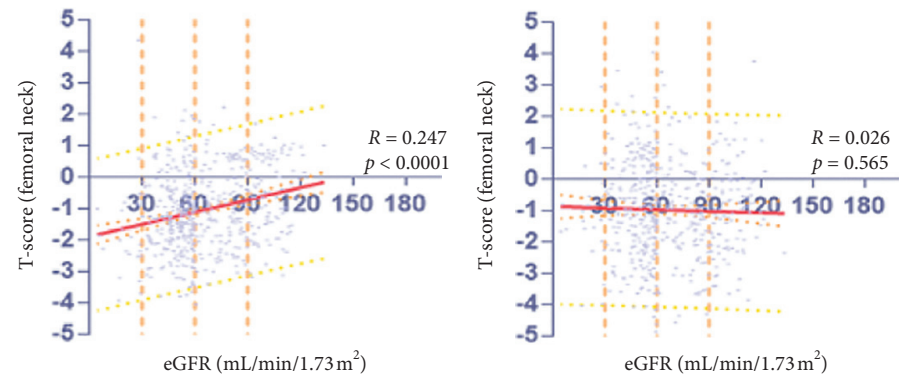

\begin{tabular}{lcccc} 
Female at-risk population & \multicolumn{2}{c}{ T-score-femoral neck } & \multicolumn{2}{c}{ T-score-lumbar spine } \\
& Coefficient & $P$ value & Coefficient & $P$ value \\
\hline Simple correlation & 0.247 & $<0.0001$ & -0.026 & 0.565 \\
Partial correlation 1 & 0.095 & 0.044 & -0.114 & 0.023 \\
Partial correlation 2 & 0.132 & 0.003 & -0.082 & 0.085 \\
Partial correlation 3 & 0.12 & 0.007 & -0.066 & 0.173 \\
\hline
\end{tabular}

(c)

FIGURe 2: Scatterplot, Pearson correlation, and partial correlations between T-score and eGFR in the total at-risk population, male at-risk population, and female at-risk population ( $\mathrm{a}, \mathrm{b}$, and $\mathrm{c}$, respectively). T-score is presented by a logarithmic scale along the $y$-axis and eGFR along the $x$-axis. Regression lines are shown with 95\% CIs (orange lines) and 95\% prediction interval (yellow lines). BMD, bone mineral density; eGFR: estimated glomerular filtration rate; the at-risk population, male participants aged $\geq 50$ years, and postmenopausal female.

3.5. Association between OP and Stage in the At-Risk CKD Population. Thereafter we investigated the relationship between OP prevalence and CKD stage in the at-risk population. Results revealed a positive correlation between OP prevalence according to FN BMD and CKD stage, whereas that according to LS BMD was not significant (Table 4). OP prevalence based on FN BMD in female or male subgroups still showed a significant association with CKD, whereas according to LS BMD it showed no significant association with $\mathrm{CKD}$ stage in either female or male subgroups.

\section{Discussion}

Our findings revealed that adjusted FN BMDs have a significant positive association with eGFR and the stage of CKD. Besides, the association is linear, depending on the stage of CKD. However, LS BMD is neither significantly associated with eGFR nor the stage of CKD. In addition, prevalence of OP in FN, but not LS BMD, showed a positive association with the stage of CKD.

Our results further indicated that CKD prevalence causes a significantly lower FN BMD, but not LS BMD. Unadjusted FN BMD was positively related to eGFR and stage of $\mathrm{CKD}$, and the relationship was still statistically significant after adjusting for multiple other confounders. However, the relationship between LS BMD and eGFR or stage of CKD was not significant after adjusting. These findings overrule accuracy of assuming a simple positive or negative relationship between eGFR and BMD, owing to an effect of potential factors such as aging process, lifestyle, medical conditions, and different sites of bone. However, these findings were partially consistent with a previous study [30]. Besides, after grouping by sex and adjusting for other confounders, we found that the association was still significant in males, but not females. A similar result was reported by Ishani et al. who found a significant association between eGFR and BMD in males [47]. This inconsistency 
TABLE 4: Prevalence of osteoporosis in the at-risk population according to the severity of CKD.

\begin{tabular}{|c|c|c|c|c|c|}
\hline & Stage I $(N=196)$ & Stage II $(N=295)$ & Stage III $(N=560)$ & Stage IV $(N=53)$ & $P$ for trend \\
\hline \multicolumn{6}{|l|}{ Osteoporosis-femoral neck ( $n, \%)$} \\
\hline Total population & $6(3.1 \%)$ & $19(6.4 \%)$ & $46(8.2 \%)$ & $9(17.0 \%)^{\dagger}$ & 0.002 \\
\hline Female & $4(4.1 \%)$ & $16(12.2 \%)$ & $36(14.2 \%)^{\dagger}$ & $5(21.7 \%)^{\dagger}$ & 0.008 \\
\hline Male & $2(2.0 \%)$ & $3(1.8 \%)$ & $10(3.3 \%)$ & $4(13.3 \%)^{\dagger}$ & 0.037 \\
\hline \multicolumn{6}{|l|}{ Osteoporosis-lumbar spine (n, \%) } \\
\hline Total population & $14(7.1 \%)$ & $26(8.8 \%)$ & $54(9.6 \%)$ & $3(5.7 \%)$ & 0.549 \\
\hline Female & $10(10.3 \%)$ & $13(9.9 \%)$ & $43(16.9 \%)$ & $2(8.7 \%)$ & 0.418 \\
\hline Male & $4(4.1 \%)$ & $13(7.9 \%)$ & $11(3.6 \%)$ & $1(6.25 \%)$ & 0.798 \\
\hline
\end{tabular}

Values are $n$ (\%). $P$ for trend values by logistic regression models, and $P<0.05$ was considered statistically significant. ${ }^{\dagger} P<0.01$ by post hoc analyses, CKD stage 1 subgroup as reference; $P<0.01$ was considered statistically significant. CKD, chronic kidney disease; the at-risk population, male participants aged $\geq 50$ years, and menopause female.

may be due to other confounders that potentially exert greater impacts on females than males.

The association between unadjusted FN BMD and the stage of CKD was significantly negatively linear, whereas that of FN BMD (for participants with stages 2, 3, and 4) was significantly different in participants with stage 1 . However, after adjusting for other confounders, only the FN BMD in participants with stages 3 and 4 was significantly different from those at stage 1 . This result is consistent with previous studies in which BMD was found to decrease in stages 3-5 [21, 48, 49]. With regard to CKD progression, several hormonal and biochemical changes such as hyperphosphatemia, hypocalcemia, increased fibroblast growth factor 23 (FGF-23), and PTH levels as well as vitamin D concentration abnormalities have been suggested [50]. Occurrence of these changes in vivo may explain the above result. Previous studies have demonstrated elevated levels of circulating osteoprotegerin (OPG) and receptor activator of nuclear factor- $\mathrm{KB}$ ligand (RANKL) in OP patients [51-54]. OPG was a factor associated with aortic stiffness and cardiovascular mortality in CKD patients [55]. Additionally, excessive bone resorption contributed to hyperphosphatemia with stimulation of heterotopic mineralization such as vascular calcification [56]. In our study, we hypothesize that CKD progression led to loss of bone mass which subsequently may increase the risk of aortic calcification and cardiovascular mortality in CKD patients. It is a vicious circle. This disorder of systems biology links skeletal and kidney dysfunction to the risk of cardiovascular diseases and mortality through the CKD-MBD [57]. Future studies should therefore focus on the kidney-skeletal-cardiovascular axis which potentially plays an important role in prognosis and survival of CKD-MBD patients. Results generated from this may lead to development of an important therapeutic target for improving long-term outcomes of CKD-MBD patients.

Chen et al. reported that prevalence of spinal fractures in CKD patients was similar to the general population [58]. Our results partly support this finding. Specifically, we found eGFR to be independently associated with FN BMD and CKD, but LS BMD was not significantly associated with eGFR. The factors that influenced the higher FN BMD during CKD progression but did not influence LS BMD remain unclear. This result may be explained by the fact that the hip has more cortical bone than, which is more impaired compared to, trabecular of the bone in CKD patients [59-61].

Previous studies have drawn different conclusion regarding Asian populations [20, 31, 62]. Results from the current study differ from the aforementioned reports, because ours were based on different ethnic population. Previous studies have showed that the effect of height, weight, body composition, environmental factors, and lifestyle may all result in ethnic differences in bone mass and eGFR [36, 63-67]. Thus, evaluating association between $\mathrm{BMD}$ and other factors based on different populations may reveal different results. Levey et al. demonstrated that the Chronic Kidney Disease Epidemiology Collaboration (CKD-EPI) equation overcomes some of the limitations of the Modification of Diet in Renal Disease (MDRD) equation and therefore has important implications for public health and clinical practice [68]. In the last decade, CKD-EPI equation was extensively used to calculate eGFR. However, recently, no studies have recently used this new equation to describe the association between eGFR and BMD in American CKD groups. It is, therefore, necessary to update our knowledge regarding the CKD-EPI equation. Generally, our study is more suitable for US populations and follows the recent advances in this domain.

The present study had some limitations. Firstly, we used a cross-sectional design in the study, which may have caused the direction of causality not to be formally assessed. Secondly, the CKD-EPI equation incorporated age, sex, and race in its calculation, which may all have impacted the BMD. It is, therefore, possible that entry of these variables may alter the association between CKD and other variables or create interaction terms in multivariate models. Furthermore, there may be a little imprecise by using eGFR, calculating by CKD-EPI equation, to represent GRF. However, to data, CKD-EPI equation remains the most accurate and widely used method for estimating GFR. Thirdly, we lacked data for hormones and markers involved in bone metabolism. Therefore, pathophysiology underlying the association between eGFR of CKD patients and BMD of different sites could not be elucidated, and, as a result, we could not evaluate the type of bone impairment. In future, prospective controlled trials should be conducted to reveal the underlying pathophysiological mechanisms and causal 
factors involved in different bone sites during CKD progression. Fourthly, our study was based on an American population, which does not represent the entire CKD population worldwide. Fifthly, we did not consider LS and FN status, in terms of presence of CKD and individual radiographic features, which might confound the BMD results. Finally, selection bias may be present due to dropped data and the nonresponse rate.

\section{Conclusion}

In summary, we assessed the association between BMD and eGFR in patients with CKD. We found that FN BMD was decreased in CKD patients. Further analysis showed that FN BMD was significantly positively associated with eGFR or stage of CKD, but there was no significant association between LS BMD and eGFR or stage of CKD. After adjusting for potential confounders, the association between FN BMD and severity of CKD persisted. These results may explain why the prevalence of hip fracture is much higher than that of spine fracture among patients with CKD. This is important as it offers a guide for effective care, prevention, and treatment of patients with CKD. However, because this was a cross-sectional study, the strength of our results is limited. Nevertheless, our results provide new ideas further to understand why FN BMD is much lower than LS BMD in patients with CKD.

\begin{tabular}{|c|c|}
\hline OP: & osteoporosis \\
\hline CKD-MBD: & $\begin{array}{l}\text { chronic kidney disease-mineral and bone } \\
\text { disorder }\end{array}$ \\
\hline KDOQI: & $\begin{array}{l}\text { The National Kidney Foundation's Kidney } \\
\text { Disease Outcomes Quality Initiative }\end{array}$ \\
\hline DXA: & dual-energy X-ray absorptiometry \\
\hline ALP: & alkaline phosphatase \\
\hline GLM: & General linear models \\
\hline FGF-23: & fibroblast growth factor 23 \\
\hline OPG: & osteoprotegerin \\
\hline RANKL: & receptor activator of nuclear factor- $\kappa \mathrm{B}$ ligand \\
\hline CKD-EPI: & $\begin{array}{l}\text { Chronic Kidney Disease Epidemiology } \\
\text { Collaboration }\end{array}$ \\
\hline FN: & femoral neck \\
\hline LS: & lumbar spine \\
\hline BMD: & bone mineral density \\
\hline CKD: & chronic kidney disease \\
\hline NHANES: & The National Health and Nutrition \\
\hline eGFR: & $\begin{array}{l}\text { Examination Survey } \\
\text { estimated glomerular filtration rate }\end{array}$ \\
\hline
\end{tabular}

\section{Data Availability}

Data are available upon reasonable request.

\section{Conflicts of Interest}

All authors declare that there are no conflicts of interest related to the present article.

\section{Authors' Contributions}

JFH, XLZ, and AMW designed the study. JFH and XQZ developed and tested the data collection forms. JFH, XQZ, XLS, XZ, JL, and AMW acquired the data. JFH, XQZ, XLS, $\mathrm{XZ}$, JL, YML, XYW, and AMW conducted the analysis and interpreted the data. JFH, XQZ, and AMW drafted the manuscript. All authors critically revised the manuscript. AiMin $\mathrm{Wu}, \mathrm{MD}, \mathrm{PhD}$, and Xiao-Lei Zhang, $\mathrm{MD}, \mathrm{PhD}$, have contributed equally to this work.

\section{Acknowledgments}

This work was supported by the National Natural Science Foundation of China (31600769 and 81501933), Wenzhou Municipal Science and Technology Bureau (Y20190018), Wenzhou Leading Talent Innovative Project (RX2016004), Zhejiang Provincial Medical Technology Foundation of China (2018KY129), Higher Education Teaching Reform Project of Wenzhou Medical University (YBJG201826), and Zhejiang Provincial Traditional Chinese Medicine Science and Technology Program (2020ZB146).

\section{References}

[1] J. Coresh, E. Selvin, L. A. Stevens et al., "Prevalence of chronic kidney disease in the United States," JAMA, vol. 298, no. 17, pp. 2038-2047, 2007.

[2] V. Jha, G. Garcia-Garcia, K. Iseki et al., "Chronic kidney disease: global dimension and perspectives," The Lancet, vol. 382, no. 9888, pp. 260-272, 2013.

[3] T. K. Chen, D. H. Knicely, and M. E. Grams, "Chronic kidney disease diagnosis and management: a review," JAMA, vol. 322, no. 13 , pp. 1294-1304, 2019.

[4] A. S. Levey, K.-U. Eckardt, Y. Tsukamoto et al., "Definition and classification of chronic kidney disease: a position statement from Kidney Disease: improving Global Outcomes (KDIGO)," Kidney International, vol. 67, no. 6, pp. 2089-2100, 2005.

[5] A. S. Levey, P. E. de Jong, J. Coresh et al., "The definition, classification, and prognosis of chronic kidney disease: a KDIGO controversies conference report," Kidney International, vol. 80, no. 1, pp. 17-28, 2011.

[6] K. L. Naylor, E. McArthur, W. D. Leslie et al., "The three-year incidence of fracture in chronic kidney disease," Kidney International, vol. 86, no. 4, pp. 810-818, 2014.

[7] L. F. Fried, M. L. Biggs, M. G. Shlipak et al., "Association of kidney function with incident hip fracture in older adults," Journal of the American Society of Nephrology, vol. 18, no. 1, pp. 282-286, 2007.

[8] "Consensus development conference: diagnosis, prophylaxis, and treatment of osteoporosis," The American Journal of Medicine, vol. 94, no. 6, pp. 646-650, 1993.

[9] J. E. Compston, M. R. McClung, and W. D. Leslie, "Osteoporosis," The Lancet, vol. 393, no. 10169, pp. 364-376, 2019.

[10] T.-L. Yang, H. Shen, A. Liu et al., "A road map for understanding molecular and genetic determinants of osteoporosis," Nature Reviews Endocrinology, vol. 16, no. 2, p. 91, 2019.

[11] M. G. Vervloet, Z. A. Massy, V. M. Brandenburg et al., "Bone: a new endocrine organ at the heart of chronic kidney disease and mineral and bone disorders," The Lancet Diabetes \& Endocrinology, vol. 2, no. 5, pp. 427-436, 2014. 
[12] A. C. Webster, E. V. Nagler, R. L. Morton, and P. Masson, "Chronic kidney disease," The Lancet, vol. 389, no. 10075, pp. 1238-1252, 2017.

[13] M. Cozzolino and M. Ketteler, "Evaluating extended-release calcifediol as a treatment option for chronic kidney diseasemineral and bone disorder (CKD-MBD)," Expert Opinion on Pharmacotherapy, vol. 20, no. 17, pp. 2081-2093, 2019.

[14] S. Moe, T. Drüeke, J. Cunningham et al., "Definition, evaluation, and classification of renal osteodystrophy: a position statement from Kidney Disease: improving Global Outcomes (KDIGO)," Kidney International, vol. 69, no. 11, pp. 1945-1953, 2006.

[15] Kidney Disease: Improving Global Outcomes (KDIGO) CKD-MBD Work Group, "KDIGO clinical practice guideline for the diagnosis, evaluation, prevention, and treatment of chronic kidney disease-mineral and bone disorder (CKDMBD)," Kidney International, no. 113, pp. S1-S130, 2009.

[16] S. M. Kim, J. Long, M. Montez-Rath, M. Leonard, and G. M. Chertow, "Hip fracture in patients with non-dialysisrequiring chronic kidney disease," Journal of Bone and Mineral Research, vol. 31, no. 10, pp. 1803-1809, 2016.

[17] National Kidney Foundation, "K/DOQI clinical practice guidelines for bone metabolism and disease in chronic kidney disease," American Journal of Kidney Diseases: The Official Journal of the National Kidney Foundation, vol. 42, pp. S1S201, 2003.

[18] T. L. Nickolas, D. J. McMahon, and E. Shane, "Relationship between moderate to severe kidney disease and hip fracture in the United States," Journal of the American Society of $\mathrm{Ne}$ phrology, vol. 17, no. 11, pp. 3223-3232, 2006.

[19] P. T. Goldenstein, S. A. Jamal, and R. M. A. Moysés, "Fractures in chronic kidney disease: pursuing the best screening and management," Current Opinion in Nephrology and Hypertension, vol. 24, no. 4, pp. 1-323, 2015.

[20] S.-W. Choi, H.-Y. Kim, H.-R. Ahn et al., "Association of bone mineral density with albuminuria and estimated glomerular filtration rate: the dong-gu study," Kidney and Blood Pressure Research, vol. 37, no. 2-3, pp. 132-141, 2013.

[21] S. K. Jassal, D. von Muhlen, and E. Barrett-Connor, "Measures of renal function, BMD, bone loss, and osteoporotic fracture in older adults: the Rancho Bernardo study," Journal of Bone and Mineral Research, vol. 22, no. 2, pp. 203-210, 2007.

[22] C.-Y. Hsu, S. R. Cummings, C. E. McCulloch, and G. M. Chertow, "Bone mineral density is not diminished by mild to moderate chronic renal insufficiency," Kidney International, vol. 61, no. 5, pp. 1814-1820, 2002.

[23] Y. S. Jung, H.-j. Hwang, B. H. Yun et al., "Renal function is associated with bone mineral density and arterial stiffness in healthy postmenopausal women," Gynecologic and Obstetric Investigation, vol. 78, no. 2, pp. 124-129, 2014.

[24] K. Uhlig, J. S. Berns, B. Kestenbaum et al., "KDOQI US commentary on the 2009 KDIGO clinical practice guideline for the diagnosis, evaluation, and treatment of CKD-mineral and bone disorder (CKD-MBD)," American Journal of Kidney Diseases, vol. 55, no. 5, pp. 773-799, 2010.

[25] T. Isakova, T. L. Nickolas, M. Denburg et al., "KDOQI US commentary on the 2017 KDIGO clinical practice guideline update for the diagnosis, evaluation, prevention, and treatment of chronic kidney disease-mineral and bone disorder (CKD-MBD)," American Journal of Kidney Diseases, vol. 70, no. 6, pp. 737-751, 2017.

[26] R. H. Yenchek, J. H. Ix, M. G. Shlipak et al., "Bone mineral density and fracture risk in older individuals with CKD,"
Clinical Journal of the American Society of Nephrology, vol. 7, no. 7, pp. 1130-1136, 2012.

[27] M. Boyle, J. P. Buckley, and L. Quirós-Alcalá, “Associations between urinary organophosphate ester metabolites and measures of adiposity among U.S. children and adults: NHANES 2013-2014," Environment International, vol. 127, pp. 754-763, 2019.

[28] G. Zipf, M. Chiappa, K. S. Porter, Y. Ostchega, B. G. Lewis, and J. Dostal, "National health and nutrition examination survey: plan and operations, 1999-2010," Vital Health Statistics, vol. 1, no. 56, pp. 1-37, 2013.

[29] J. T. Schousboe, J. A. Shepherd, J. P. Bilezikian, and S. Baim, "Executive summary of the 2013 international society for clinical densitometry position development conference on bone densitometry," Journal of Clinical Densitometry, vol. 16, no. 4, pp. 455-466, 2013.

[30] K. S. Bezerra de Carvalho, R. F. V. Vasco, M. R. Custodio, V. Jorgetti, R. M. A. Moysés, and R. M. Elias, "Chronic kidney disease is associated with low BMD at the hip but not at the spine," Osteoporosis International, vol. 30, no. 5, pp. 1015-1023, 2019.

[31] H.-L. Kim, I. Y. Park, J. M. Choi et al., "A decline in renal function is associated with loss of bone mass in Korean postmenopausal women with mild renal dysfunction," Journal of Korean Medical Science, vol. 26, no. 3, pp. 392-398, 2011.

[32] Centers for Disease Control and Prevention National Center for Health Statistics, Dual Energy X-Ray Absorptiometry (DXA) Procedures Manual, 2007, http://www.cdc.gov/nchs/ data/nhanes/nhanes_07_08/manual_dexa.pdf.

[33] A. C. Looker, N. Sarafrazi Isfahani, B. Fan, and J. A. Shepherd, "Trends in osteoporosis and low bone mass in older US adults, 2005-2006 through 2013-2014," Osteoporosis International, vol. 28, no. 6, pp. 1979-1988, 2017.

[34] J. M. Wishart, A. O. Need, M. Horowitz, H. A. Morris, and B. E. C. Nordin, "Effect of age on bone density and bone turnover in men," Clinical Endocrinology, vol. 42, no. 2, pp. 141-146, 1995.

[35] G. A. Greendale, M. Sowers, W. Han et al., "Bone mineral density loss in relation to the final menstrual period in a multiethnic cohort: results from the Study of Women's Health across the Nation (SWAN)," Journal of Bone and Mineral Research, vol. 27, no. 1, pp. 111-118, 2012.

[36] A. C. Looker, H. W. Wahner, W. L. Dunn et al., "Updated data on proximal femur bone mineral levels of US adults," Osteoporosis International, vol. 8, no. 5, pp. 468-490, 1998.

[37] World Health Organization, "Assessment of fracture risk and its application to screening for postmenopausal osteoporosis," Technical report series no. 843, WHO, Geneva, Switzerland, 1994.

[38] J. A. Kanis, L. J. Melton 3rd, C. Christiansen, C. C. Johnston, and N. Khaltaev, "The diagnosis of osteoporosis," Journal of Bone and Mineral Research, vol. 9, no. 8, pp. 1137-1141, 1994.

[39] A. S. Levey, A. Levin, and J. A. Kellum, "Definition and classification of kidney diseases," American Journal of Kidney Diseases, vol. 61, no. 5, pp. 686-688, 2013.

[40] M. T. Mefford, R. S. Rosenson, L. Deng et al., "Trends in statin use among US adults with chronic kidney disease, 1999-2014," Journal of the American Heart Association, vol. 8, no. 2, Article ID e010640, 2019.

[41] D. Murphy, C. E. McCulloch, F. Lin et al., "Trends in prevalence of chronic kidney disease in the United States," Annals of Internal Medicine, vol. 165, no. 7, pp. 473-481, 2016. 
[42] S. Klawansky, E. Komaroff, P. F. Cavanaugh Jr. et al., "Relationship between age, renal function and bone mineral density in the US population," Osteoporosis International, vol. 14, no. 7, pp. 570-576, 2003.

[43] J. Bover, P. Ureña-Torres, A. M. Laiz Alonso et al., "Osteoporosis, bone mineral density and CKD-MBD (II): therapeutic implications," Nefrología, vol. 39, no. 3, pp. 227-242, 2019.

[44] P. Khairallah and T. L. Nickolas, "Updates in CKD-associated osteoporosis," Current Osteoporosis Reports, vol. 16, no. 6, pp. 712-723, 2018.

[45] B.-L. Pan and S.-S. Loke, "Chronic kidney disease associated with decreased bone mineral density, uric acid and metabolic syndrome," PLoS One, vol. 13, no. 1, Article ID e0190985, 2018.

[46] A. K. Akobeng, "Understanding type I and type II errors, statistical power and sample size," Acta Paediatrica, vol. 105, no. 6, pp. 605-609, 2016.

[47] A. Ishani, M. Paudel, M. Paudel et al., "Osteoporotic fractures in men study $\mathrm{G}$ (2008) renal function and rate of hip bone loss in older men: the osteoporotic fractures in men study," Osteoporosis International, vol. 19, no. 11, pp. 1549-1556, 2008.

[48] A. Levin, G. L. Bakris, M. Molitch et al., "Prevalence of abnormal serum vitamin $\mathrm{D}, \mathrm{PTH}$, calcium, and phosphorus in patients with chronic kidney disease: results of the study to evaluate early kidney disease," Kidney International, vol. 71, no. 1, pp. 31-38, 2007.

[49] L. Malmgren, F. McGuigan, A. Christensson, and K. E. Akesson, "Reduced kidney function is associated with $\mathrm{BMD}$, bone loss and markers of mineral homeostasis in older women: a 10-year longitudinal study," Osteoporosis International, vol. 28, no. 12, pp. 3463-3473, 2017.

[50] A. Covic, M. Vervloet, Z. A. Massy et al., "Bone and mineral disorders in chronic kidney disease: implications for cardiovascular health and ageing in the general population," The Lancet Diabetes \& Endocrinology, vol. 6, no. 4, pp. 319-331, 2018.

[51] N. Morabito, A. Gaudio, A. Lasco et al., "Osteoprotegerin and RANKL in the pathogenesis of thalassemia-induced osteoporosis: new pieces of the puzzle," Journal of Bone and Mineral Research, vol. 19, no. 5, 2004.

[52] Y. Chiba, T. Onouchi, T. Ikeda, J. Adachi, Y. Tamura, and T. Horiuchi, "Implications of measuring soluble receptor activators of nuclear factor-kappaB ligand and osteoprotegerin in bone metabolism of elderly women," Gerontology, vol. 55, no. 3, 2009.

[53] I. Nabipour, B. Larijani, K. Vahdat et al., "Relationships among serum receptor of nuclear factor-kappaB ligand, osteoprotegerin, high-sensitivity C-reactive protein, and bone mineral density in postmenopausal women: osteoimmunity versus osteoinflammatory," Menopause, vol. 16, no. 5, 2009.

[54] S. Jabbar, J. Drury, J. N. Fordham, H. K. Datta, R. M. Francis, and S. P. Tuck, "Osteoprotegerin, RANKL and bone turnover in postmenopausal osteoporosis," Journal of Clinical $\mathrm{Pa}$ thology, vol. 64, no. 4, 2011.

[55] M. L. Ford, E. R. Smith, L. A. Tomlinson, P. K. Chatterjee, C. Rajkumar, and S. G. Holt, "FGF-23 and osteoprotegerin are independently associated with myocardial damage in chronic kidney disease stages 3 and 4 . Another link between chronic kidney disease-mineral bone disorder and the heart," $\mathrm{Ne}$ phrology, Dialysis, Transplantation, vol. 27, no. 2, 2012.

[56] S. Mathew, K. S. Tustison, T. Sugatani, L. R. Chaudhary, L. Rifas, and K. A. Hruska, "The mechanism of phosphorus as a cardiovascular risk factor in CKD," Journal of the American Society of Nephrology, vol. 19, no. 6, 2008.

[57] K. A. Hruska, T. Sugatani, O. Agapova, and Y. Fang, "The chronic kidney disease-mineral bone disorder (CKD-MBD): advances in pathophysiology," Bone, vol. 100, pp. 80-86, 2017.

[58] H. Chen, P. Lips, M. G. Vervloet, N. M. van Schoor, and R. T. de Jongh, "Association of renal function with bone mineral density and fracture risk in the Longitudinal Aging Study Amsterdam," Osteoporosis International, vol. 29, no. 9, pp. 2129-2138, 2018.

[59] R. K. Hall, R. Sloane, C. Pieper et al., "Competing risks of fracture and death in older adults with chronic kidney disease," Journal of the American Geriatrics Society, vol. 66, no. 3, pp. 532-538, 2018.

[60] J. Przedlacki, J. Buczyńska-Chyl, J. Buczyńska-Chyl et al., “The utility of FRAX in predicting bone fractures in patients with chronic kidney disease on hemodialysis: a two-year prospective multicenter cohort study," Osteoporosis International, vol. 29, no. 5, pp. 1105-1115, 2018.

[61] D. Matuszkiewicz-Rowińska, J. B. Olesen, G. H. Gislason, B. Abrahamsen, and K. Hommel, "Risk of fracture in adults on renal replacement therapy: a Danish national cohort study," Nephrology Dialysis Transplantation, vol. 31, no. 10, pp. 1654-1662, 2016.

[62] J.-P. Myong, H.-R. Kim, J.-W. Koo, and C. Y. Park, "Relationship between bone mineral density and moderate to severe chronic kidney disease among general population in Korea," Journal of Korean Medical Science, vol. 28, no. 4, pp. 569-574, 2013.

[63] S. Silverman, A. Calderon, K. Kaw et al., "Patient weighting of osteoporosis medication attributes across racial and ethnic groups: a study of osteoporosis medication preferences using conjoint analysis," Osteoporosis International, vol. 24, no. 7, pp. 2067-2077, 2013.

[64] S. Matsuo, Y. Yasuda, E. Imai, and M. Horio, "Current status of estimated glomerular filtration rate (eGFR) equations for Asians and an approach to create a common eGFR equation," Nephrology, vol. 15, no. 2, pp. 45-48, 2010.

[65] K. Kin, J. H. Lee Edmund, K. Kushida et al., "Bone density and body composition on the Pacific rim: a comparison between Japan-born and U.S.-born Japanese-American women," Journal of Bone and Mineral Research, vol. 8, no. 7, pp. 861-869, 1993.

[66] E. Barrett-Connor, E. S. Siris, L. E. Wehren et al., "Osteoporosis and fracture risk in women of different ethnic groups," Journal of Bone and Mineral Research, vol. 20, no. 2, pp. 185-194, 2005.

[67] S. Andersen, E. Boeskov, and P. Laurberg, "Ethnic differences in bone mineral density between inuit and caucasians in North Greenland are caused by differences in body size," Journal of Clinical Densitometry, vol. 8, no. 4, pp. 409-414, 2005.

[68] A. S. Levey, L. A. Stevens, C. H. Schmid et al., "A new equation to estimate glomerular filtration rate," Annals of Internal Medicine, vol. 150, no. 9, pp. 604-612, 2009. 